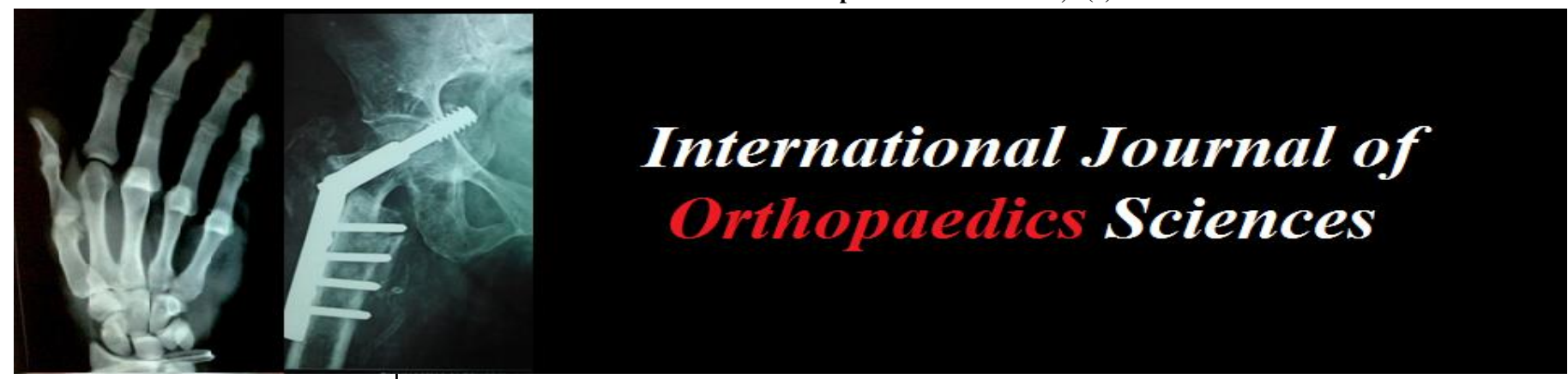

E-ISSN: 2395-1958

P-ISSN: 2706-6630

IJOS 2020; 6(2): 611-617

(C) 2020 IJOS

www.orthopaper.com

Received: 19-02-2020

Accepted: 21-03-2020

Sushant S Ghumare

B.J. Govt. Medical College \&

Sassoon General Hospital, Pune,

Maharashtra, India

Ajay S Chandanwale

B.J. Govt. Medical College \&

Sassoon General Hospital, Pune,

Maharashtra, India

Pradeep Jadhav

B.J. Govt. Medical College \&

Sassoon General Hospital, Pune,

Maharashtra, India

Samarth Arya

Wrightington Hospital, WWL

NHS Foundation Trust, Wigan,

United Kingdom

Surendra Singh Rawat

Wrightington Hospital, WWL

NHS Foundation Trust, Wigan,

United Kingdom

Dr. Suraj Sankar

Wrightington Hospital

WWL NHS Foundation

Trust, Wigan, United

Kingdom
Corresponding Author: Surendra Singh Rawat

Wrightington Hospital, WWL

NHS Foundation Trust, Wigan,

United Kingdom

\section{A study of relationship between vitamin b12 levels and neurological function in patients with cervical spondylotic myelopathy}

\author{
Sushant S Ghumare, Ajay S Chandanwale, Pradeep Jadhav, Samarth \\ Arya, Surendra Singh Rawat and Dr. Suraj Sankar
}

DOI: https://doi.org/10.22271/ortho.2020.v6.i2j.2109

\section{Abstract}

Cervical spondylotic myelopathy refers to a clinical syndrome of long tract signs in the upper and lower extremities arising from a combination of static and dynamic compression ${ }^{[1]}$. Vitamin B 12 deficiency leads to the development of degenerative disease of the peripheral and central nervous systems. There have been studies on subacute combined degeneration of the spinal cord resulting from VB12 deficiency ${ }^{[2]}$ but, no studies to confirm the role of Vitamin B12 in the pathogenesis of CSM ${ }^{[3]}$. The present study is to find out a co-relation between VB12 deficiency and CSM.

Methods: Our study includes 100 patients with CSM to evaluate for co-relation with VB12 deficiency. Demographic data, clinical, radiological and laboratory investigations were done to find a co-relation between VB12 deficiency and severity of symptoms.

Result: The level of involvement was maximum at C5-C6 level (42\%) followed by C4-C5 level (28\%) and C6-C7 levels (20\%). Sensory abnormalities were seen in $61 \%$ cases, while motor weakness was reported in 50\% cases. Prevalence of VB12 deficiency was noted in 47\% of cases. VB12 deficiency was seen in $40 \%$ cases of level 1 compression and $54.1 \%$ and $53.8 \%$ cases of level 2 and 3 cord compression respectively. No significant association was observed between level of cord compression and VB12 deficiency (p- 0.37). Significant association was seen between VB12 deficiency and sensory abnormalities $(p<0.05)$. A higher prevalence of VB12 deficiency was seen in cases with motor weakness in all four limbs (48.6\%) as compared to isolated involvement of upper or lower limbs $(33.3 \%$ and $14.3 \%)$.

Conclusion: The results in the present study suggested that the incidence of CSM may be correlated with Vitamin B 12 deficiency, particularly for cases in which the clinical manifestations and the MR imaging do not fully match.

Keywords: Cervical spondylotic myelopathy; vitamin B12 deficiency

\section{Introduction}

Cervical spondylotic myelopathy (CSM) refers to age-related degenerative changes within the cervical spinal column leading to spinal cord compression in the neck ${ }^{[1]}$. Clinical features for this myelopathy are pain and numbness in limbs, poor coordination, weakness, increased tone along with bladder problems. Due to it's mobility, cervical spine is more prone to degenerative changes as disc prolapse, ligament hypertrophy, ossification of posterior longitudinal ligament and osteophytosis and collectively these changes are called cervical spondylosis and are more common in elderly age group ${ }^{[4]}$. CSM causes progressive neurological deficits and can cause quadriplegia and wheelchair dependence ${ }^{[5]}$. Therefore, urgent treatment, preferably within six months of onset, in the form of spinal decompression is needed to halt the disease progression as delayed treatment can cause life-long disability ${ }^{[6]}$.

Vitamin B12 deficiency is caused by a lack in diet and malabsorption in the gastrointestinal tract or by genetic predisposition presenting as various hematological, gastrointestinal and neuropsychiatric manifestations like myelopathy, neuropathy, dementia and optic nerve atrophy ${ }^{[7]}$. Clinical features related to neurology for Vitamin B 12 deficiency are due to the involvement of posterior and lateral columns of cervical and upper dorsal part of spinal cord and are labelled as sub-acute combined degeneration (SACD) of spine ${ }^{[8]}$. 
The symptomatology of SACD involves dysthesia, gait disturbance with loss of position and vibration sense in lower and upper limbs, para-paresis and tetra-paresis, and it resembles clinical features of CSM ${ }^{[9]}$.

Treatment with supplementation of vitamin B12 helps in some degree of reversal in symptomatology in most patients and complete recovery in almost $50 \%$ of patients.

$\mathrm{Xu}$, Chen and Jiang reported two cases where CSM was corelated to VB 12 deficiency. They were treated with decompression and VB 12 supplementation and showed considerable improvement ${ }^{[3]}$. The pathogenesis of cervical myelopathy is not clearly understood, but overlapping clinical picture and such case reports in the literature suggest some co-relation. However, to date, there have been no studies establishing a relationship between serum VB12 levels and the severity of symptoms \& neurological deterioration in patients with CSM.

\section{Methodology}

Ethics approval: An observational cross-sectional study conducted at a tertiary care centre of B. J. Medical College and Sassoon Hospital, Pune, and approved by the regional ethical committee.

Study Area: Department of Orthopaedics, B.J. Govt. medical college \& Sassoon General Hospital, Pune

Study Population: All the patients with cervical spondylotic myelopathy, diagnosed on MRI.

\section{Study Design: Observational Study}

Sample Size Calculation: Consecutive type of nonprobability sampling was used for selection of cases. A total of 100 cases with cervical myelopathy diagnosed on MRI coming in the department of orthopaedics fulfilling inclusion and exclusion criteria were selected for the study.

\section{Inclusion Criteria}

1. Patients with degeneration of cervical intervertebral disc leading to disc bulge/protrusion/extrusion on MRI compressing the spinal cord.

2. Patients with chronic neck pain with features of cervical myelopathy such as pain, numbness, loss of sensation, loss of power and increase in tone in upper limbs.

\section{Exclusion Criteria}

Patients with diagnosed neurological dysfunction such as stroke, peripheral neuropathies, Guillain-Barre syndrome, ataxia, traumatic cervical spine injury, congenital spine deformities, fractures in the upper limb, psychological dysfunction, tuberculosis of the spine and other infections of the spinal cord.

\section{Methodology}

A detailed history was taken regarding the onset and progression of symptoms, including history of trauma. A detailed neurological examination was done and documented for comparison in the future for deterioration or improvement. $\mathrm{X}$-ray cervical spine AP and Lateral view and magnetic resonance imaging (MRI) cervical spine were done, and the degree of cord compression was documented for all patients. Degree of the spinal cord compression was classified into one of four groups as follows- Level 0 - no pressure on spinal cord, Level 1 - mild compression on the spinal cord, Level 2 - the degree of spinal cord compression is $<1 / 3$, Level 3 - the degree of spinal cord compression is $>1 / 3$.

Laboratory investigations- complete blood count, random blood sugar level, vitamin B12 levels (normal range: 211 $946 \mathrm{pg} / \mathrm{l}$ ), and RA factor were done and recorded.

\section{Statistical Analysis}

The quantitative data was represented as their mean \pm SD. Categorical and nominal data were expressed in percentage. Results of VB12 Levels were compared statistically with severity of symptoms and cord compression. The t-test was used for analysing quantitative data, or else non -parametric data was analysed by Mann Whitney test, and categorical data was analysed by using chi-square test. The significance threshold of p-value was set at $<0.05$. All analysis was carried out by using SPSS software version 21.

\section{Observations \& Results}

The mean age of the study cases was 59.32 years, with $73 \%$ cases were above 50 years of age. (Figure 1). Male predominance was seen in the present study with $61 \%$ males to $39 \%$ females (Figure 2) the level of involvement was maximum at C5-C6 level (42\%) followed by C4-C5 level (28\%) and C6-C7 levels (20\%) (Figure 3). Sensory abnormalities were seen in $61 \%$ cases, while motor weakness was reported in 50\% cases (Figure 4). Among 50 cases with motor weakness, the involvement of all four limbs was reported in 37 cases, while isolated upper and lower limb involvement was seen in 6 and 7 cases respectively (Figure $5)$. The level of cord compression was observed on MRI. Level 1 cord compression was seen in 50\% cases, while level 2 and level 3 cord compression was observed in $37 \%$ and $13 \%$ cases respectively (Figure 6). Prevalence of Vitamin B12 deficiency was noted in $47 \%$ of cases of cervical spondylotic myelopathy. Vitamin B12 deficiency was seen in $40 \%$ cases of level 1 compression and $54.1 \%$ and $53.8 \%$ cases of level 2 and 3 cord compression respectively (Figure 7). No significant association was observed between the level of cord compression and vitamin B12 deficiency (p-0.37)

(Figure 8). A significant association was seen between the prevalence of vitamin B12 deficiency and sensory abnormalities $(p<0.05)$ (Figure 9).

\section{Discussion}

A hospital-based observational study was conducted to establish a relationship between serum VB12 levels and severity of symptoms \& neurological deterioration in patients with CSM. Consecutive type of non-probability sampling was used for the selection of cases. A total of 100 cases of cervical myelopathy diagnosed on MRI coming in the orthopaedics department of our hospital and fulfilling inclusion criteria were selected for the study.

Demography: The mean age of the study cases was 59.32 years, with $73 \%$ cases were above 50 years of age. Similar findings were seen in the study by Zhu et al. (2013), with average age of 49.54 (32-69) years. Male predominance was seen in the present study, with $61 \%$ males to $39 \%$ females. The study conducted by Kumar et al. of the 25 patients also had male predominance with 23 were male ${ }^{[10]}$.

Level Of Involvement: Level of involvement was maximum at C5-C6 level (42\%) followed by C4-C5 level (28\%) and C6C7 levels (20\%). In the study by Chagas et al., the most common level involved was $\mathrm{C} 5-\mathrm{C} 6$ of $71.7 \%$. This shows that 
C5-C6 level of cervical myelopathy is the most common level of involvement ${ }^{[11]}$.

Symptoms: Sensory abnormalities were seen in $61 \%$ cases, while motor weakness was reported in $50 \%$ cases. Bladder involvement was seen in $7 \%$ of cases. Among 50 cases with motor weakness, involvement of all four limbs was reported in 37 cases, while isolated upper and lower limb involvement was seen in 6 and 7 cases respectively. Similar findings were seen in the studies by Zhu et al ${ }^{[12]}$.

Cord Compression Levels: MRI cervical spine was done to evaluate the degree of the spinal cord compression. In the present study, level 1 cord compression was seen in 50\% cases, while level 2 and level 3 cord compression was observed in $37 \%$ and $13 \%$ cases respectively.

Vitamin B12 Levels: In the present study, the prevalence of VB12 deficiency was noted in $47 \%$ of cases of CSM. VB12 deficiency was seen in $40 \%$ cases of level 1 compression and $54.1 \%$ and $53.8 \%$ cases of level 2 and 3 cord compression respectively. No significant association was observed between level of cord compression and VB12 deficiency (p-0.37). A significant association was seen between the prevalence of VB12 deficiency and sensory abnormalities $(p<0.05)$. Also, a higher prevalence of VB12 deficiency was seen in cases with motor weakness in all four limbs $(48.6 \%)$ as compared to isolated involvement of upper or lower limbs $(33.3 \%$ and $14.3 \%)$. Xu et al, described two cases with clinical features of cervical myelopathy and low level of Vitamin B 12 but with not much changes in MRI. The authors observed improvement following cervical decompression surgery and VB12 replacement therapy. The features of CSM in these patients could be attributed to lack of VB12 [3]. There have been various published case reports for reversible myelopathy changes, responding to VB12 replacement therapy ${ }^{[8]}$. Therefore, it is recommended that the serum VB12 levels are checked in cases of CSM where the MR imaging does not show stenotic changes. At present, there is still a lack of investigation into the role of $\mathrm{VB} 12$ in the pathogenesis of CSM. Further studies are recommended to investigate the incidence of VB12 deficiency in patients with CSM.

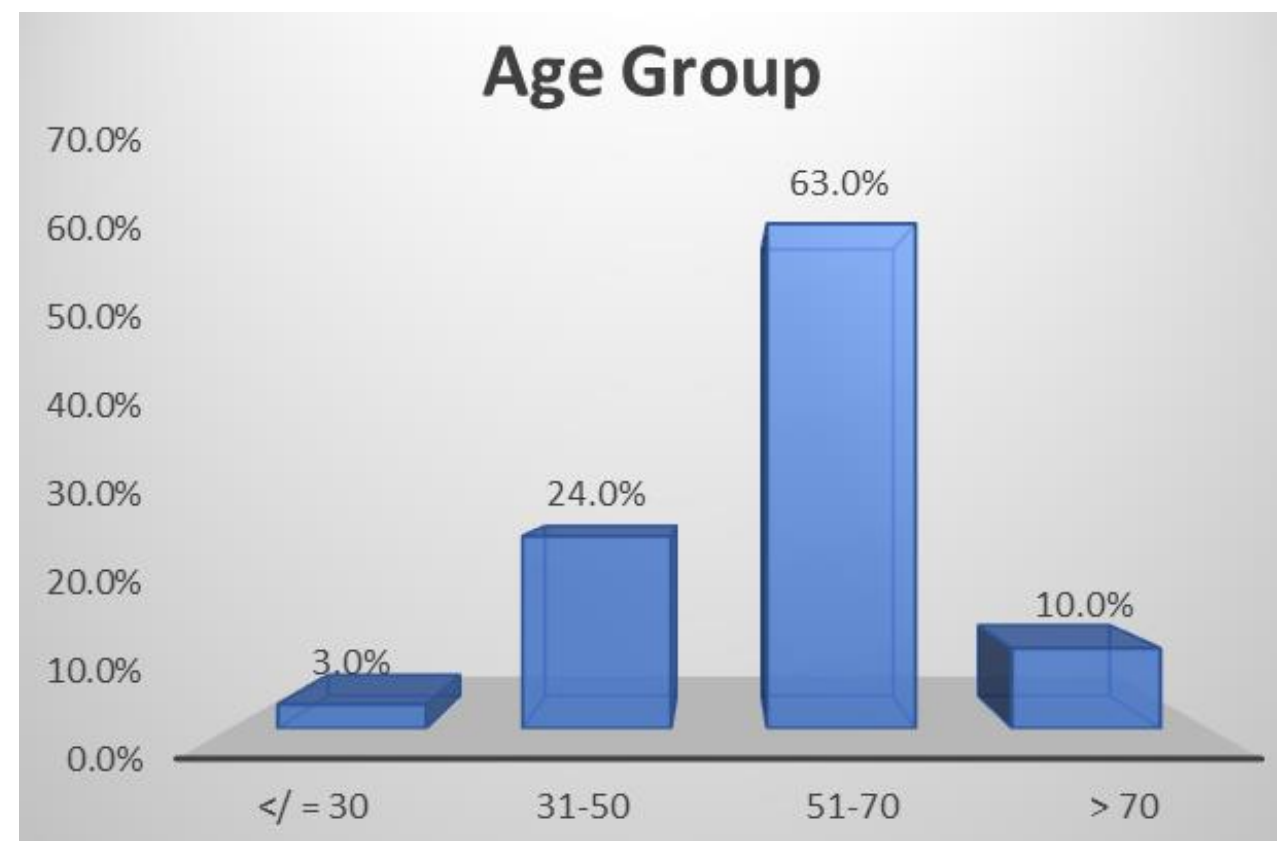

Fig 1: The mean age of the study cases was 59.32 years, with $73 \%$ cases were above 50 years of age.

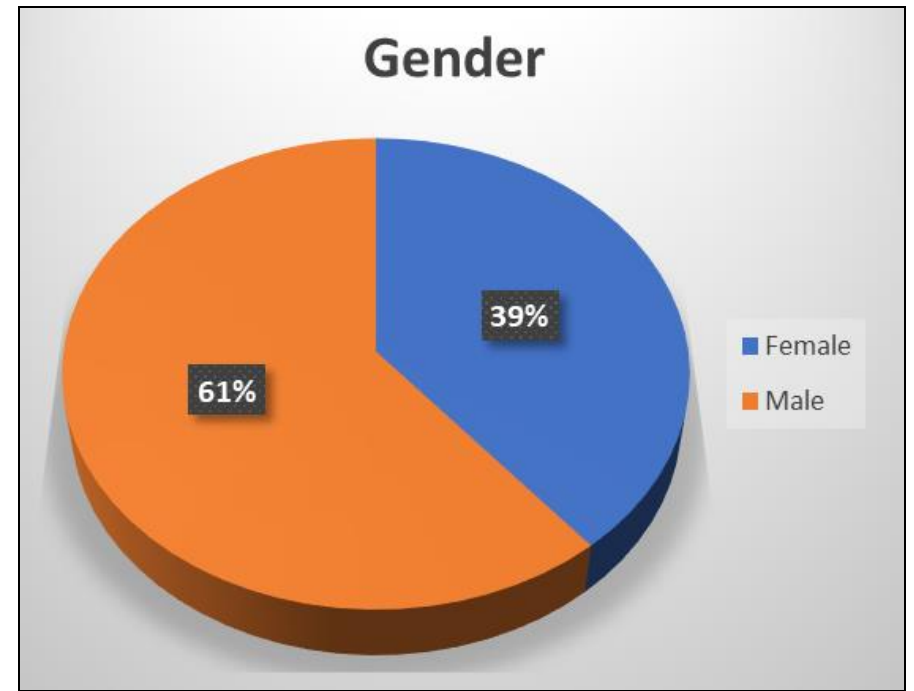

Fig 2: Male predominance was seen in the present study with $61 \%$ males to $39 \%$ females 


\section{Level of Involvement}

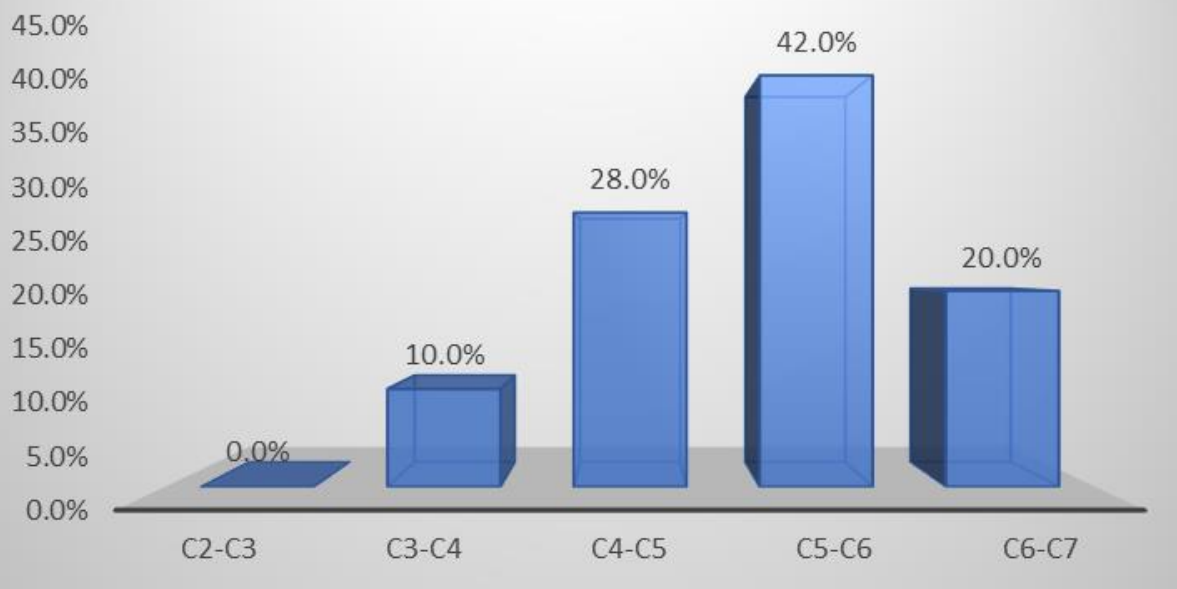

Fig 3: The level of involvement was maximum at C5-C6 level (42\%) followed by C4-C5 level (28\%) and C6-C7 levels (20\%).

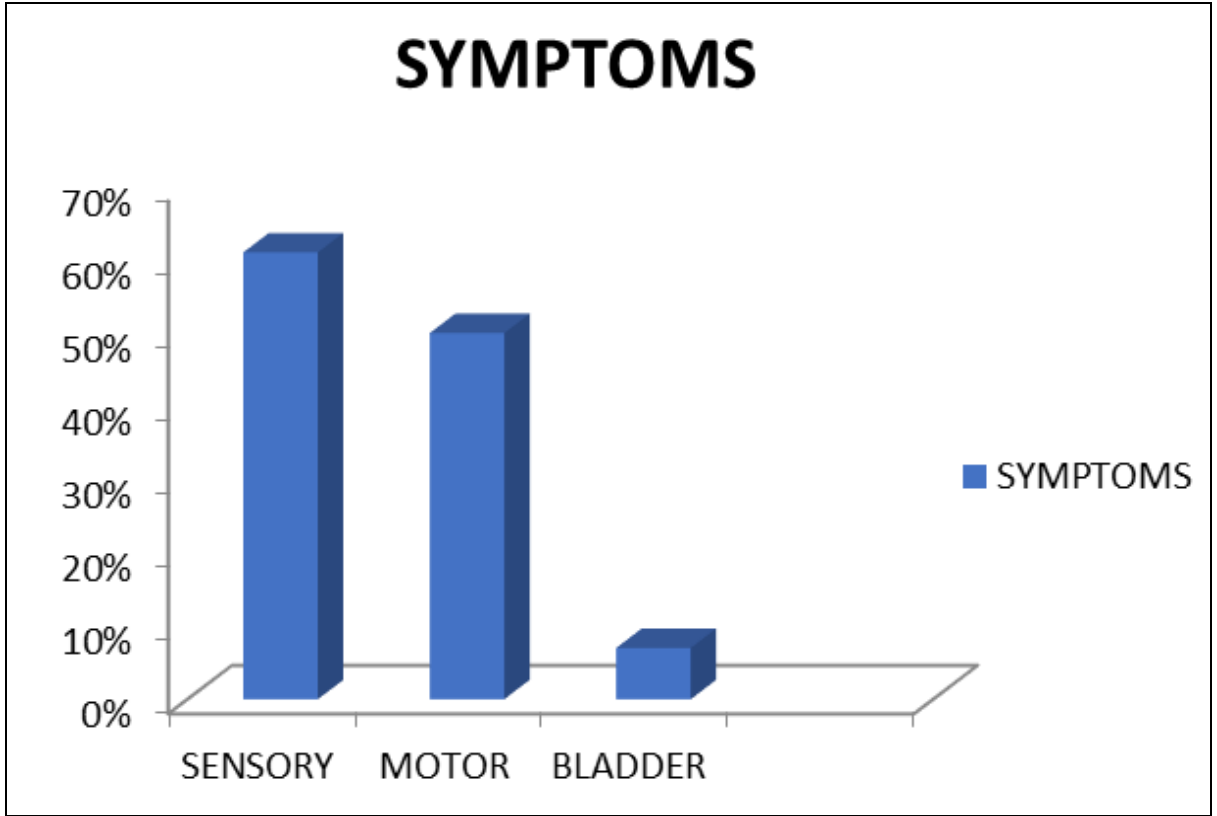

Fig 4: Sensory abnormalities were seen in $61 \%$ cases, while motor weakness was reported in $50 \%$ cases.

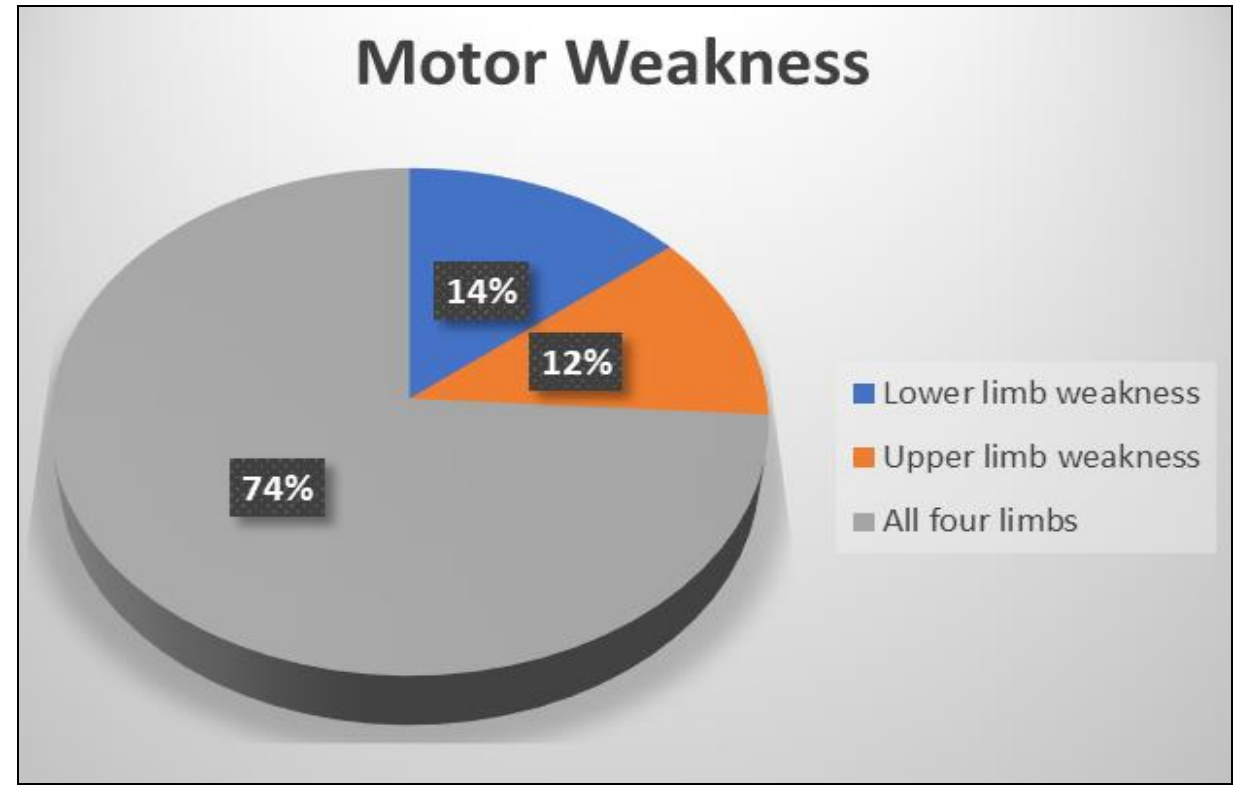

Fig 5: Among 50 cases with motor weakness, the involvement of all four limbs was reported in 37 cases, while isolated upper and lower limb involvement was seen in 6 and 7 cases respectively 


\section{Cord Compression}

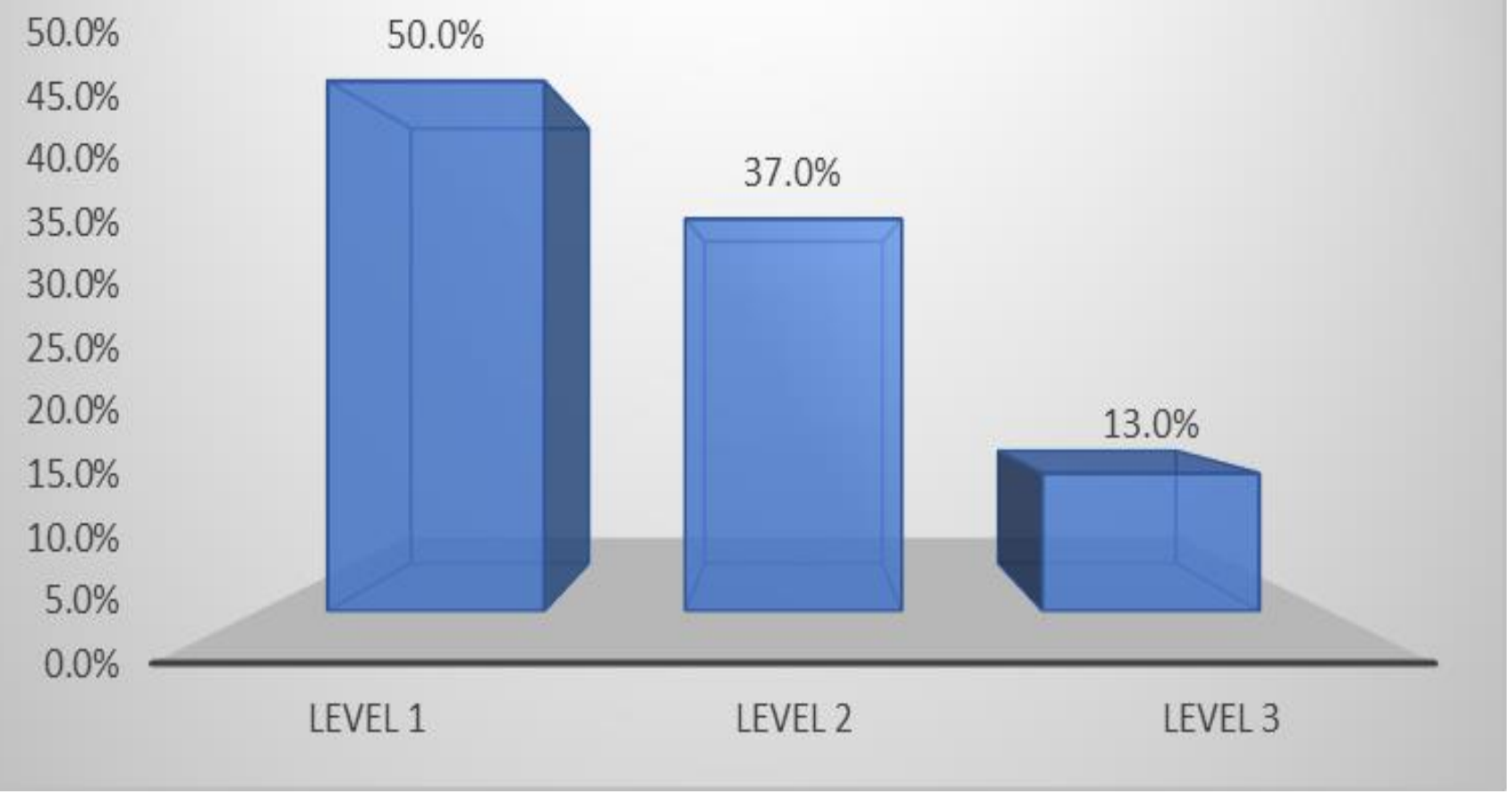

Fig 6: The level of cord compression was observed on MRI. Level 1 cord compression was seen in 50\% cases, while level 2 and level 3 cord compression was observed in $37 \%$ and $13 \%$ cases respectively.

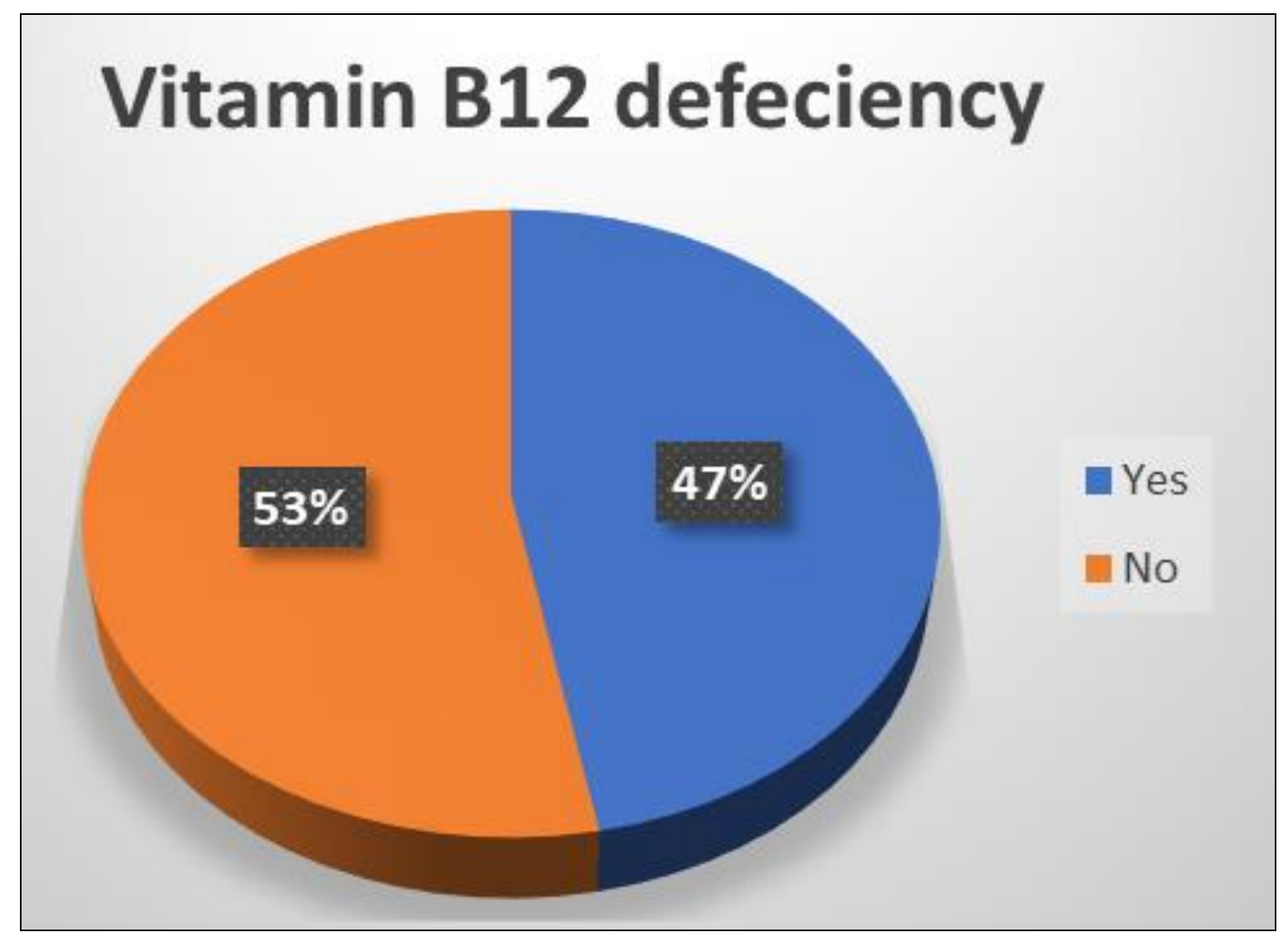

Fig 7: Prevalence of Vitamin B12 deficiency was noted in 47\% of cases of cervical spondylotic myelopathy. 


\section{Cord Compression vs Vitamin B12 \\ defeciency}

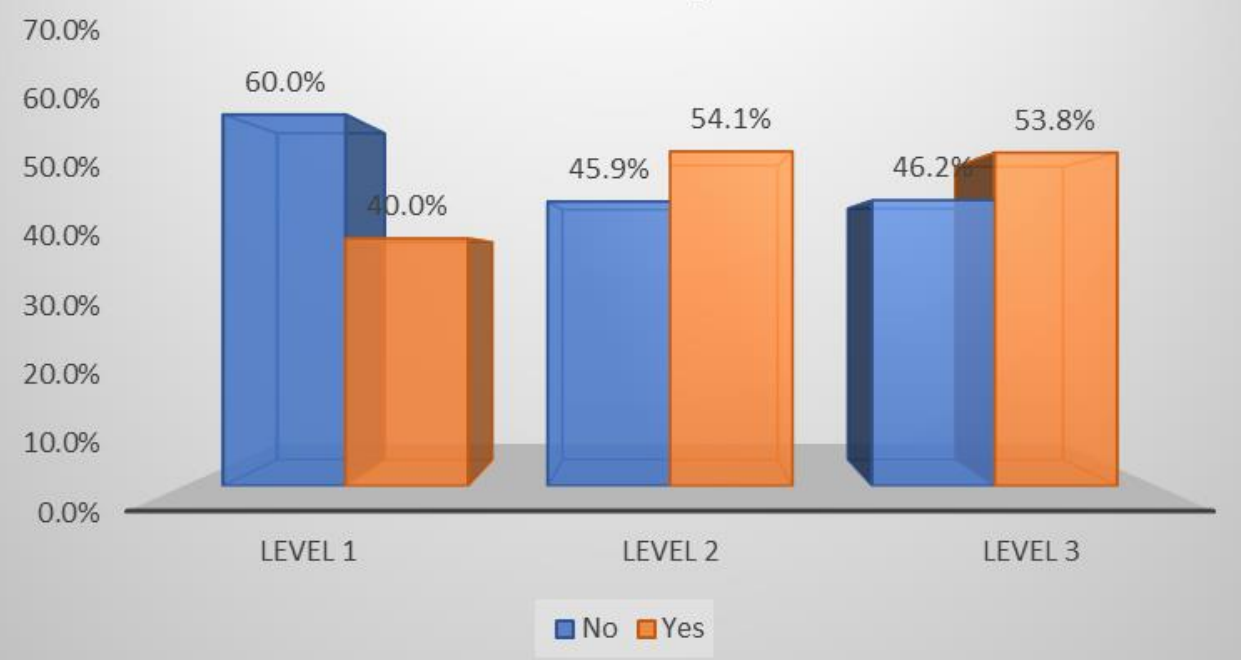

Fig 8: Vitamin B12 deficiency was seen in $40 \%$ cases of level 1 compression and $54.1 \%$ and $53.8 \%$ cases of level 2 and 3 cord compression respectively. No significant association was observed between the level of cord compression and vitamin B12 deficiency (p-0.37).

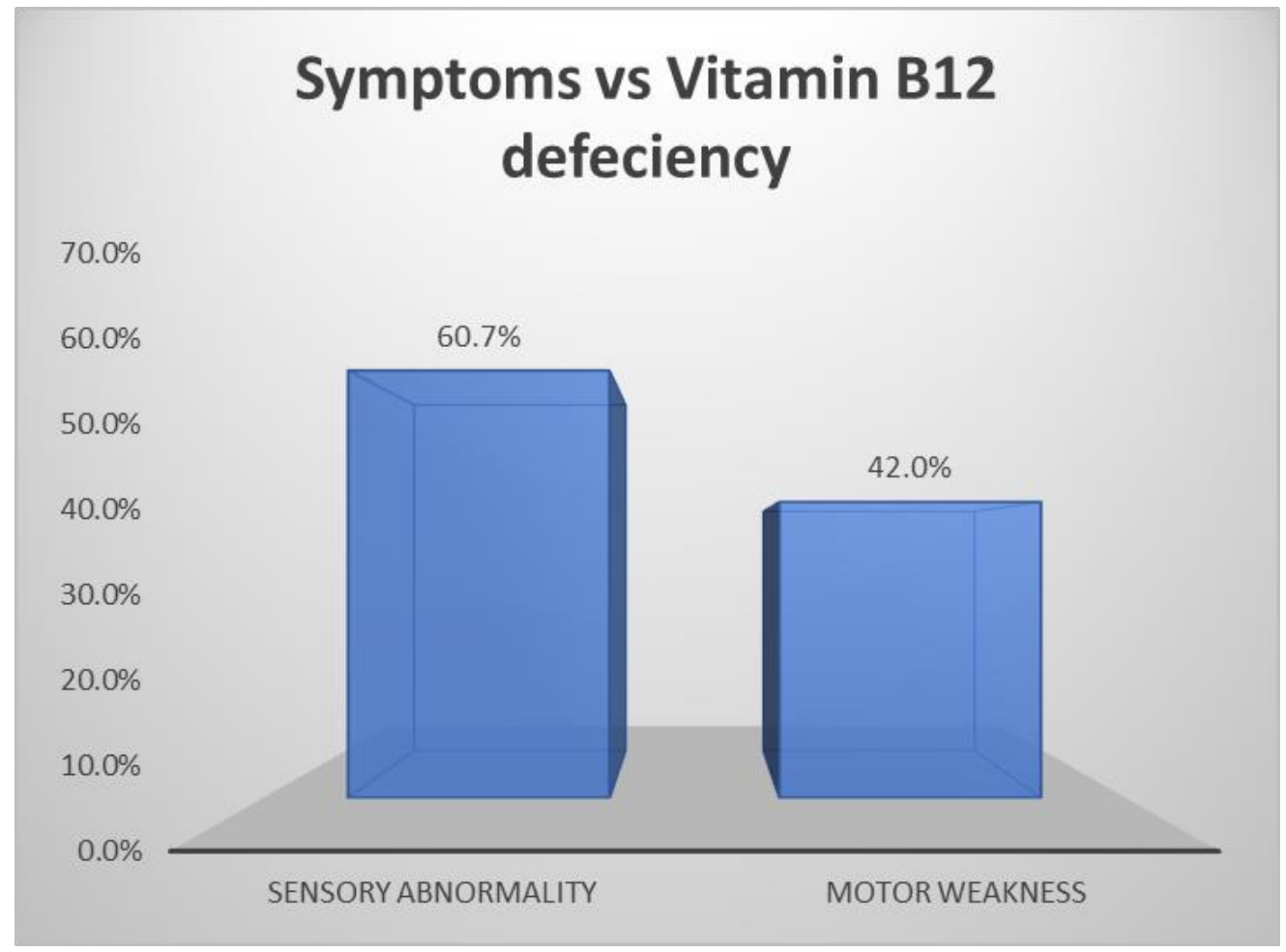

Fig 9: A significant association was seen between the prevalence of vitamin B12 deficiency and sensory abnormalities $(p<0.05)$.

\section{Conclusion}

In conclusion, the results in the present study suggest that cervical spinal myelopathy may be correlated with VB 12 deficiency, particularly in cases where MRI does not give a clear picture. Association of VB12 deficiency and CSM suggest for routine assessment of levels in the blood. It would be prudent to further investigate whether patients with both CSM and VB12 deficiency present differently clinically or if they have sub-optimal results after treatment or whether VB12 supplementation gives better results. Further studies in this area would be easily feasible because VB12 is an essential vitamin, cheap, and readily available.

\section{Acknowledgments}

This research did not receive financial support from funding agencies in the public, commercial, or not-for-profit sectors.

\section{References}

1. Davies B, Mowforth O, Smith E, Kotter M. Degenerative cervical myelopathy. BMJ. 2018, k186.

2. Nouri A, Patel K, Montejo J, Nasser R, Gimbel D, Sciubba D et al. The Role of Vitamin B12 in the Management and Optimization of Treatment in Patients With Degenerative Cervical Myelopathy. Global Spine Journal. 2018; 9(3):331-337.

3. XU Y, CHEN W, JIANG J. Cervical spondylotic 
myelopathy with vitamin B12 deficiency: Two case reports. Experimental and Therapeutic Medicine. 2013; 6(4):943-946.

4. Kovalova I, Kerkovsky M, Kadanka Z, Kadanka Z, Nemec M, Jurova B et al. Prevalence and Imaging Characteristics of Nonmyelopathic and Myelopathic Spondylotic Cervical Cord Compression. SPINE. 2016; 41(24):1908-1916.

5. Oh T, Lafage R, Lafage V, Protopsaltis T, Challier V, Shaffrey C et al. Comparing Quality of Life in Cervical Spondylotic Myelopathy with Other Chronic Debilitating Diseases Using the Short Form Survey 36-Health Survey. World Neurosurgery. 2017; 106:699-706.

6. Tetreault L, Côté P, Kopjar B, Arnold P, Fehlings M. A clinical prediction model to assess surgical outcome in patients with cervical spondylotic myelopathy: internal and external validations using the prospective multicenter AOSpine North American and international datasets of 743 patients. The Spine Journal. 2015; 15(3):388-397.

7. Wehrmacher W, Messmore H. Book Reviews: Wintrobe's Clinical Hematology, 10th Edition. G. Richard Lee, John Foerster, John Lukens, Frixos Para skevas, John P. Greer, and George M. Rodgers, (eds.) with 86 contributors. Williams and Wilkins, Baltimore MD, 1999, 2763 pp., Illustrated, \$179.00. Clinical and Applied Thrombosis/Hemostasis. 1999; 5(3):201-201.

8. Pittock S, Payne T, Harper C. Reversible Myelopathy in a 34-Year-Old Man with Vitamin B12 Deficiency. Mayo Clinic Proceedings. 2002; 77(3):291-294.

9. Hemmer B, Glocker F, Schumacher M, Deuschl G, Lucking C. Subacute combined degeneration: clinical, electrophysiological, and magnetic resonance imaging findings. Journal of Neurology, Neurosurgery \& Psychiatry. 1998; 65(6):822-827.

10. Kumar V, Rea G, Mervis L, McGregor J. Cervical Spondylotic Myelopathy: Functional and Radiographic Long-term Outcome after Laminectomy and Posterior Fusion. Neurosurgery. 1999; 44(4):771-777.

11. Chagas H, Domingues F, Aversa A, Vidal Fonseca A, de Souza J. Cervical spondylotic myelopathy: 10 years of prospective outcome analysis of anterior decompression and fusion. Surgical Neurology. 2005; 64:S30-S35.

12. Zhu B, Xu Y, Liu X, Liu Z, Dang G. Anterior approach versus posterior approach for the treatment of multilevel cervical spondylotic myelopathy: a systemic review and meta-analysis. European Spine Journal. 2013; 22(7):1583-1593. 